\title{
Research Progress of Traditional Chinese and Western Medicine on Polycystic Ovary Syndrome
}

\author{
Lu Zhang1*, Hongli Zhu ${ }^{2}$ \\ 1Shaanxi University of Traditional Chinese Medicine, Xianyang 712046, Shaanxi Province, China \\ 2Department of Gynecology, Affiliated Hospital of Shaanxi University of Traditional Chinese Medicine, Xianyang 712046, \\ Shaanxi Province, China \\ *Corresponding author: Lu Zhang, fkxs0715@163.com

\begin{abstract}
Polycystic ovary syndrome (PCOS) is a disease with reproductive dysfunction and abnormal endocrine metabolism. It is characterized by hyperandrogenism, insulin resistance (IR) and persistent anovulation. At present, the pathogenesis and pathological mechanism of PCOS are still unclear, and there is no unified standard for the diagnosis and treatment of PCOS locally nor abroad. It is still a famous and difficult problem to explore the pathogenesis of PCOS and seek effective prevention and treatment methods.
\end{abstract}

Keywords: Polycystic ovary syndrome; Western medicine; Chinese medicine; overview

Publication date: July 2021; Online publication: July 31, 2021

\section{Progress in western medicine research}

\subsection{Western medicine etiology and pathology: Obesity}

In recent years, the obesity problem of PCOS has attracted increasingly more attention, as $30 \%-70 \%$ of PCOS patients are obese ${ }^{[1]}$. Additionally, it is mainly manifested in abdominal obesity. It has been reported that the incidence of abdominal obesity is as high as $80 \%$. Some scholars believe that polyphagia during adolescence, familial inheritance or high androgen uterine environment during pregnancy may lead to obesity and PCOS in adulthood ${ }^{[2]}$. Obesity and PCOS are mutually causal. Obesity in young and middleage women can cause menstrual abnormalities and sparse ovulation, which in turn promotes the occurrence of PCOS, or makes obese PCOS patients cause more severe hyperandrogenemia, insulin resistance and other reproductive endocrine disorders.

The incidence of ovarian dysfunction is significantly higher in obese PCOS patients than in non-obese PCOS patients ${ }^{[3]}$. Androgen (T), luteinizing hormone/follicle stimulating hormone (LH/FSH), insulin resistance (IR), fasting blood glucose and insulin water in obese PCOS patients are basically higher than those in non-obese PCOS patients. western medicine studies have shown that obesity is positively related to IR. Adipose tissue secretes a large number of chronic inflammatory factors, such as interleukin- 6 and tumor necrosis factor- $\alpha$, which make the insulin signaling pathway abnormal and cause IR. The risk of long-term complications of obese patients with insulin resistance in PCOS patients has almost increased exponentially. Therefore, it is very necessary for obese PCOS patients to take active measures to reduce BMI and improve IR.

\subsection{Western medicine etiology and pathology: Chronic inflammatory factors}

In 2001, Kelly et al. ${ }^{[4]}$ found that the body of PCOS patients was in a sub-clinical inflammatory response state for the first time in 2001. This sub-clinical response state is the so-called chronic low-grade 
inflammation, which is mainly reflected in interleukin 6 (IL-6), tumor necrosis factor $\alpha$ (TNF- $\alpha$ ), transforming growth factor- $\beta 1$ (TGF- $\beta 1$ ), C-reactive protein (CRP), plasma plasminogen activator inhibitor 1 (PAI-1), monocyte chemotaxis. The increase of chemical factor 1 (MCP-1) and other acute phase reaction products. Chronic low-grade inflammation can cause disorders of ovarian function in patients, promote the occurrence and development of PCOS, and have a great relationship with the metabolic abnormalities of PCOS patients ${ }^{[5]}$. Relevant studies in recent years ${ }^{[6-7]}$ show that obesity itself is a sub-clinical inflammatory state, effecting the adipose tissue. The levels of secreted various inflammatory factors such as CRP, IL-6, TNF- $\alpha$, MCP-1, TGF- $\beta 1$, etc. in PCOS patients are significantly higher than those in normal people. This shows that the level of sub-clinical chronic inflammatory factors is elevated. It is also one of the important pathological links of PCOS.

\subsection{Western medicine etiology and pathology: Intestinal microecological disorders}

In 2012, Pearce et al. proposed that long-term bad eating habits can lead to the imbalance of the intestinal flora, and the disordered intestinal flora damages the intestinal mucosal barrier function and causes the lipopolysaccharide (LPS) of gram-negative bacteria to enter the systemic circulation ${ }^{[8]}$. After LPS enters the systemic circulation, it activates the human immune system and causes chronic inflammation. The longterm existence of chronic inflammation can interfere with the function of insulin receptors, causing pancreatic islet $\mathrm{P}$ cells to secrete more insulin, trying to balance the increasing level of insulin in human serum, causing insulin resistance. In turn, the increase in androgen synthesis affects the normal development of follicles, and ultimately leads to the occurrence of PCOS. Therefore, disturbance of intestinal flora may be one of the causes of polycystic ovary syndrome and insulin resistance.

\section{Western medicine treatment}

\subsection{Improve lifestyle}

Improvement of lifestyle and lifestyle intervention are listed as the first-line treatment of PCOS in China and abroad ${ }^{[9]}$. The main purpose of lifestyle intervention is to control patient weight and gain muscle. The main methods include diet control, exercise, behavior intervention and other methods. First, the diet advocates low-sugar, high-fiber (low-calorie), and unsaturated fatty acid diets. At the same time, it is necessary to change past bad eating habits. In terms of exercise, you need to maintain an appropriate amount of regular energy-consuming exercise, control the daily exercise time to 30 minutes, and exercise at least five times a week to reduce sedentary behavior. Behavioral intervention is mainly to change the living habits and mental state that are prone to cause diseases under the guidance of clinicians and other relevant professional teams, thus traditional diet control or exercise measures are more effective. The Cupisti's study found that after the weight of obese PCOS patients was reduced by 5\% compared with the previous period, about $89 \%$ of the patients could return to regular menstruation, and $30 \%$ of the patients could get pregnant spontaneously. At the same time, the patients' blood lipids, insulin and androgen levels will also be improved ${ }^{[10]}$.

\subsection{Medication}

Temporary contraceptives, such as Diane-35, can treat hyperandrogenemia in PCOS patients. Oral temporary contraceptives can effectively regulate the menstrual cycle of PCOS patients, at the same time can prevent endometrial hyperplasia and improve the performance of patients with high androgen. Insulin sensitizers are used to treat insulin resistance and hyperinsulinemia, mainly by reducing insulin levels in patients, improving insulin sensitivity, and reducing androgen levels in the body to help patients restore menstrual cycles and normal ovulation. A large number of existing studies have proved that insulin sensitizers also have a good weight loss effect. 
In recent years, many scholars in China and abroad have proposed metformin as the first-line treatment for PCOS. Metformin is currently the most common drug used to improve insulin resistance. Of course, for PCOS patients with fertility requirements, after the basic treatment, some patients can return to normal ovulation and become pregnant, but most patients still need further ovulation induction therapy. Letrozole has become the first-line treatment of PCOS-type infertility drugs ${ }^{[9]}$. Letrozole blocks the production of estrogen, relieves the negative feedback inhibition effect of estrogen on the HPO axis, causes the secretion of endogenous Gonadotropins ( $\mathrm{Gn}$ ) to increase and promote follicular development. It also prevents the conversion of androgens to estrogen, and increases androgen levels. Elevate and accumulate in the follicle, increase the expression of FSH receptor, and promote the growth and development of the follicle. Existing research results show that, the use of letrozole to promote ovulation, the patient's ovulation rate, single follicular development rate and fetal live birth rate are better than clomiphene, and the risk of multiple pregnancy is relatively small.

\subsection{Surgical treatment}

Laparoscopic ovarian perforation is often used. Surgical treatment can improve the local blood circulation of the patient's ovaries, increase the level of gonadotropins, and reduce the level of estrogen in the serum, which can help restore the patient's ovarian function to a certain extent. However, surgical treatment is a traumatic treatment, and there may be problems such as ineffective treatment, pelvic adhesions, and ovarian insufficiency. Therefore, surgical treatment is not recommended as a routine.

\section{Research progress of TCM}

\subsection{The etiology and pathogenesis of TCM}

Traditional Chinese medicine (TCM) does not have a disease name that accurately corresponds to PCOS. According to its clinical symptoms and characteristics, the disease is classified as "amenorrhea," irregular menstruation," and "infertility." TCM believes that the kidney store's the essence and governs reproduction, and the kidney is closely related to the reproduction of men and women. The "Huangdi Neijing" has a more detailed record of the relationship between kidney, menstruation and reproduction. "Suwen · Strange Disease Theory" says, "The cell network is tied to the kidney." The kidney directly provides the material basis for menstruation and fetal pregnancy. Just as "Fu Qingzhu Nvke" mentioned that, "The water flows out of the kidneys." On the other hand, the spleen governs transportation and chemistry, has the characteristics of promoting clearness and lowering turbidity, preferring dryness and avoiding dampness, and is the foundation of acquired nature and the source of Qi and blood biochemistry. Insufficiency of the kidney Yang, "the fire does not warm the soil," the spleen loses its vitality, accumulates dampness and produces phlegm, blocks Chong and Ren, and if the cell network is blocked, the menstrual flow will stop. Just as "Jingyue Quanshu · Phlegm Drink" says, "phlegm is water, its origin is in the kidney, and its mark is in the spleen." "Danxi Heart Method" said, "Fatty women with irregular menstrual flow cannot become pregnant; Phlegm and dampness can block the uterus." In the third aspect, "Ye Tianshi" pointed out that, "Women are born with the liver. The liver's functions of storing blood and cleansing are also closely related to menstruation and reproduction." "Fu Qingzhu Nvke" said, "Women who have PCOS related problems and cannot give birth, is due to the liver Qi stagnation." It is not difficult to see that the etiology and pathogenesis of the disease are the dysfunction of the kidney, spleen, and liver, as well as phlegm and dampness. The existence of pathological products such as blood stasis, etc., makes any uterine axis dysfunction in Tianguiyi Chong. 


\section{Syndrome differentiation and treatment}

\subsection{TCM Treatment}

Many literatures and studies have shown that TCM for the treatment of PCOS are mainly based on drugs for invigorating the kidney, soothing the liver, strengthening the spleen, resolving phlegm, and promoting blood circulation and blood stasis. It actually varies from person to person, plus or minus according to the diagnosis. Feng Liping, ${ }^{[11]}$ believed that the main pathogenesis of PCOS is kidney deficiency and blood stasis through many years of literature, and medications are mostly based on these two aspects. Wang Yan, Shi Dongqing, mentions that through clinical studies, it has been shown that TCM for soothing the liver and regulating Qi can effectively regulate the levels of sex hormones and endocrine in patients ${ }^{[12]}$. Ye Liqun ${ }^{[13]}$ and other studies have concluded that the simultaneous application of TCM for strengthening the spleen and expectorant and catgut embedding at acupoints has a significant effect on the glucose and lipid metabolism of patients.

\section{Other treatments}

\subsection{Acupuncture therapy}

Acupuncture has been used to treat menstrual disorders, infertility, and obesity caused by PCOS since the 1980s. due to its advantages of simplicity, economy, safety and effectiveness, it has been welcomed by patients. It has achieved good effects in improving PCOS endocrine and metabolic disorders, ovulation induction, and has a good effect on improving the insulin sensitivity of patients ${ }^{[14]}$. Ren Lina's ${ }^{[15]}$ statistical analysis of most of the research results shows that acupuncture can increase the pregnancy rate and reduce the patient's HOMA-IR and BMI values.

\subsection{Acupoint massage}

Acupoint massage mainly uses point-kneading, pushing, rubbing, rubbing and other techniques. Shenshu, Pishu, Mingmen, Fenglong, Huaroumen, Tianshu, Zusanli, Sanyinjiao and other acupoints are often selected. Zhang Hesong ${ }^{[16]}$ showed that exercise combined with massage intervention in severely obese adolescents can effectively reduce body weight, BMI, and body fat rate. Luo Xiangqun ${ }^{[17]}$ and other studies have shown that Dachaihu decoction combined with kidney meridian massage can effectively reduce serum levels of hormones such as LH, T and LH/FSH. Guo Yin ${ }^{[18]}$ and others showed that after aerobic exercise combined with local massage, the serum fatty acid content of patients increased, the Monounsaturated fatty acids significantly increased, and the adipose triglyceride lipase in the cytoplasm of the patient's fat cells was significantly increased, thereby destroying the fat cells to achieve the purpose of promoting the health of the patients.

\subsection{Acupoint embedding}

Catgut embedding at acupoints is to pierce the absorbable catgut into the subcutaneous fat layer or deep into the muscle layer through a needle, so that the irritant produces a continuous, benign and gentle "permanent needle sensation" on the acupoints to achieve an effective treatment. According to the research in recent years, it is concluded that the top 10 acupoints with acupoint catgut implantation in the treatment of polycystic ovary syndrome are, Tianshu, Guanyuan, Fenglong, Taimai, Sanyinjiao, Shenshu, Zhongwan, Zusanli, Qihai, Yinling and Spring ${ }^{[19]}$. Most of these points are concentrated in the "Spleen Channel," "Stomach Channel" and "Ren Channel." Catgut embedding at acupoints regulates and controls the patient's diet, improves bowel movements, enhances energy consumption, and accelerates the decomposition of the intestine by interfering with the patient's nervous system ${ }^{[20]}$. Yan Dajing ${ }^{[21]}$ said that nutritional therapy combined with acupoint catgut implantation can improve the glucose metabolism, lipid metabolism and peripheral hormone levels of obese PCOS patients, thereby increasing the ovulation rate and pregnancy 
rate. Gao Lei found through experiments that catgut embedding at acupoints achieves the purpose of suppressing appetite and reducing weight by increasing the content of obestainin in the body. Zhao Xixin ${ }^{[23]}$ pointed out that catgut embedding at acupoints can strengthen fat metabolism, increase the sensitivity of leptin receptors and insulin receptors, thereby inhibiting people's feeding behavior and regulating fat metabolism.

\subsection{Abdominal acupuncture treatment}

Shao Zhiying selected acupoints such as Guanyuan, Zhongwan, waterway, Qi and blood, sea of Qi, lower wrist, intermediate, and lower rheumatism on the clinical curative effect of patients with PCOS infertility. He believed that the abdomen points were selected for daily treatment. One time, treatment for 3 days, rule out menstrual period, better effect is desirable ${ }^{[24]}$.

\section{Summary}

PCOS is a common reproductive endocrine disorder in women of childbearing age. The incidence rate of women of childbearing age is $5 \%$ to $10 \%$, and there is a huge group of infertile patients. Therefore, it has received increasing attention from patients and scholars. A large number of literature records and research shows that Western medicine and TCM have their own very effective programs and treatment methods in the treatment of polycystic ovary syndrome. Each has its own shortcomings.

Western medicine is still controversial and needs to be studied in the application and treatment of certain drugs. For example, the effect of metformin on weight loss in obese PCOS patients is still controversial. Although bariatric surgery for the treatment of obese PCOS has been reported, the best surgical method is still in the clinical exploration stage.

Although TCM treatment is highly safe, it has a long period of time and lacks evidence-based medicine and pharmacological support. It is basically based on doctors' experience to use drugs, which will greatly limit the development of TCM.

Therefore, the research on PCOS still needs continuous efforts. We should draw on the strengths of TCM and western medicine, combine TCM and Western medicine, and give full attention to the advantages of integrated TCM and Western medicine in the treatment of PCOS, and seek a better way for patients with PCOS. Safe, non-invasive and effective treatment method.

\section{Funding}

Social development science and technology project: 2016SF-362 Clinical study on the effect of electroacupuncture combined with walking through meridians on body mass index, glucose metabolism and reproductive endocrine in patients with obese polycystic ovary syndrome.

\section{Disclosure statement}

The author declares no conflict of interest.

\section{References}

[1] Zhang C, Shen T, 2012, Treatment of 30 Cases of Infertility with Polycystic Ovary Syndrome by Staged Medicine Cake Moxibustion and Acupuncture. Chinese Acupuncture, 32(01): 42

[2] He Y, Xu X, Li W, Yao L, et al., 2015, Research Progress in Traditional Chinese and Western Medicine for Obese Polycystic Ovary Syndrome. Chinese Journal of Traditional Chinese Medicine, 33(07): 1614-1616. 
[3] He Y, Peng Z, Han C, et al., 2015, A Clinical Study of Low-Carbohydrate Diet on Improving Infertility in Obese Patients with Polycystic Ovary Syndrome. Chinese Journal of Family Planning, 23(04) :235-238.

[4] Kelly CC, Lyall H, Petrie JR, et al., 2001, Low Grade Chronic Inflammation in Women with Polycystic Ovary Syndrome. J Clin Endocrinol Metab, 86 (6): 2453-2455.

[5] Spfitzer PM, Lecke SB, Sader F, et al., 2015, Adipose Tissue Dysfunction, Adipokines, and LowGrade Chronic Iilflammation in Polycystic Ovary Syndrome. Reproduction, 149(5): R219 R227. DOI: 10.1530/REP. 14-0435.

[6] Escobar-Morreale HF, Luque-Ramírez M, Gonzalez F, 2011, Circulating Inflammatory Markers in Polycystic Ovary Syndrome: A Systematic Review and Meta Analysis. Fertil Steril, 95(3):10481058.

[7] Atabekoglu CS, Sonmezer M, Ozmen B, et al., 2011, Increased Monocyte Chemoattractant Protein-1 Levels Indicating Early Vascular Damage in Lean Young PCOS Patients. Fertil Steril, 95(1):295-297.

[8] Yang Y, 2019, The Role and Mechanism of Intestinal Flora in the Occurrence of Polycystic Ovary Syndrome and Insulin Resistance. Southern Medical University.

[9] 2020, Consensus on Infertility Treatment and Fertility Protection Related to Polycystic Ovary Syndrome, Journal of Reproductive Medicine, 29 (7): 843-851

[10] Lei X, 2014, A Clinical Study on the Treatment of Polycystic Ovary Syndrome with Obesity and Hyperinsulinemia with Integrated Traditional Chinese and Western Medicine. Chengdu University of Traditional Chinese Medicine.

[11] Feng L, Pan W, 2016, Research on Polycystic Ovary Syndrome TCM Syndromes and Medication Rules in the Past 5 Years. Clinical Journal of Traditional Chinese Medicine, 28 (7): 955-958)

[12] Wang H, Cheng L, Ding Y, et al., 2018, Jilin Traditional Chinese Medicine. 38(12).

[13] Ye L, Yang Z, Jiang Y, et al., 2018, The Effect of Combined Treatment of Spleen-Invigorating and Expectorant Chinese Medicine and Acupoint Embedding on Glucose and Lipid Metabolism in Obese Polycystic Ovary Syndrome. China Journal of Traditional Chinese Medicine, 36(07): 16341636.

[14] Meng J, 2011, Clinical and Experimental Research on the Treatment of PCOS Insulin Resistance by Lingshu Granules. Guangzhou University of Traditional Chinese Medicine.

[15] Ren L, 2014, Acupuncture Treatment of Polycystic Ovary Syndrome: A Systematic Review and Meta-Analysis of Randomized Controlled Trials. Beijing University of Traditional Chinese Medicine.

[16] Zhang H, 2016, Analysis of the Application Effect of Exercise Combined with Massage in the Weight Loss of Severely Obese Adolescents. China Health Industry, 13(05): 68-70.

[17] Luo X, Lin J, Chen X, et al., 2017, A Randomized Controlled Study on Treatment of Obese Polycystic Ovary Syndrome with Dachaihu Decoction Combined with Kidney Meridian Massage. Chinese Maternal and Child Health Research, 28(S2):29-30.

[18] Guo Y, 2014, Research on the Effects of Exercise-Diet Intervention and Local Massage on Fat Metabolism and Weight Loss. Shanghai Institute of Physical Education.

[19] Yu C, Yao G, Zhou Y, et al., 2016, Study on the Law of Clinical Point Selection for the Treatment of Obese Polycystic Ovary Syndrome with Catgut Embedding at Acupoints. Journal of Acupuncture and Moxibustion, 32(11): 73-76. 
[20] Ye L, Yang Z, Jiang Y, et al., 2018. The Effect of Combined Treatment of Spleen-Invigorating and Expectorant Chinese Medicine and Acupoint Embedding on Glucose and Lipid Metabolism in Obese Polycystic Ovary Syndrome. China Journal of Traditional Chinese Medicine, 36(07): 16341636.

[21] Yan D, Zhang L, Zhang B, et al., 2018, Clinical Observation of Nutritional Therapy Combined with Acupoint Catgut Implantation in the Treatment of Obese Polycystic Ovary Syndrome. Clinical Misdiagnosis Governance, 31(12): 70-75.

[22] Gao Lei, Shi Xian. The Effect of Acupuncture and Catgut Embedding on Food Intake and Lipid Metabolism of Simple Obese Rats. Journal of the Academy of Continuing Medical Education, 2011, 32(6): 656-658.

[23] Zhao X, Zheng L, 2012, The Effect of Acupuncture and Catgut Embedding on Total Cholesterol, Triacylglycerol, Low Density Lipoprotein and High-Density Lipoprotein in Obese Rats. Journal of Traditional Chinese Medicine, 27 (04): 444-445.

[24] Shao Z, 2020, To Explore the Clinical Efficacy of Combined Traditional Chinese and Western Medicine in the Treatment of 88 Cases of Phlegm-Damp Type Polycystic Ovary Syndrome Infertility. Clinical Research, 28(8): 34-36. 